\title{
INTELIGENCIA ARTIFICIAL
}

\section{Education 4.0 using artificial intelligence for students performance analysis}

\author{
Zhongshan Chen ${ }^{[1], *}$, Juxiao Zhang ${ }^{[1]}$, Xiaoyan Jiang ${ }^{[1]}$, Zuojin $\mathrm{Hu}^{[1]}$, Xue $\mathrm{Han}^{[1]}$, Mengyang \\ $\mathrm{Xu}^{[2]}$, Savitha $\mathrm{V}^{[3]}$, Vivekananda $\mathrm{GN}^{[3]}$ \\ ${ }^{[1]}$ School of Mathematics and Information Science, Nanjing Normal University of Special \\ Education,Shennong Road1, Qixia District, Nanjing, China \\ ${ }^{[2]}$ College of Art and Design, Nanjing Normal University of Special Education,Shennong \\ Road1, Qixia District, Nanjing, China \\ ${ }^{[3]}$ SNS College of Technology, India \\ ${ }^{[4]}$ Madanapalle Institute of Technology and Science, India \\ *Corresponding author:Zhongshan Chen; E-mail:ycddzjb@163.com
}

\begin{abstract}
Nowadays, predicting students' performance is one of the most specific topics for learning environments, such as universities and schools, since it leads to the development of effective mechanisms that can enhance academic outcomes and avoid destruction. In education 4.0, Artificial Intelligence (AI) can play a key role in identifying new factors in students' performance and implementing personalized learning, answering routine student questions, using learning analytics, and predictive modeling. It is a new challenge to redefine education 4.0 to recognize the creative and innovative intelligent students, and it is difficult to determine students' outcomes. Hence, in this paper, Hybridized Deep Neural Network (HDNN) to predict student performance in Education 4.0. The proposed HDNN method is utilized to determine the dynamics that likely influence the student's performance. The deep neural network monitors predict, and evaluate students' performance in an education 4.0 environment. The findings show that the proposed HDNN method achieved better prediction accuracy when compared to other popular methods.
\end{abstract}

Keywords:Education 4.0, Artificial Intelligence, Students Performance Analysis.

\section{Overview of Education 4.0 using Artificial intelligence}

In education 4.0, university students' academic performance is the most significant criterion for measuring university students' performance [1]. It serves as the basic requirement for assessing teaching and learning content and evaluating and choosing university students. Today, most universities face daunting challenges in recruiting [2] new applicants because of extremely competitive educational markets. Thus, examining students' academic achievements is of high value in fostering the curriculum $[3,4]$ and enhancing the standard of education 4.0. However, student performance is determined intricately by several factors, and the socio-economic context of the student [5] and its past academic performance can theoretically impact their academic achievement [6]. Most of the current research works are limited to evaluating and forecasting students' success using mathematical methods in a relatively simple formulation of issues $[7,8]$. 


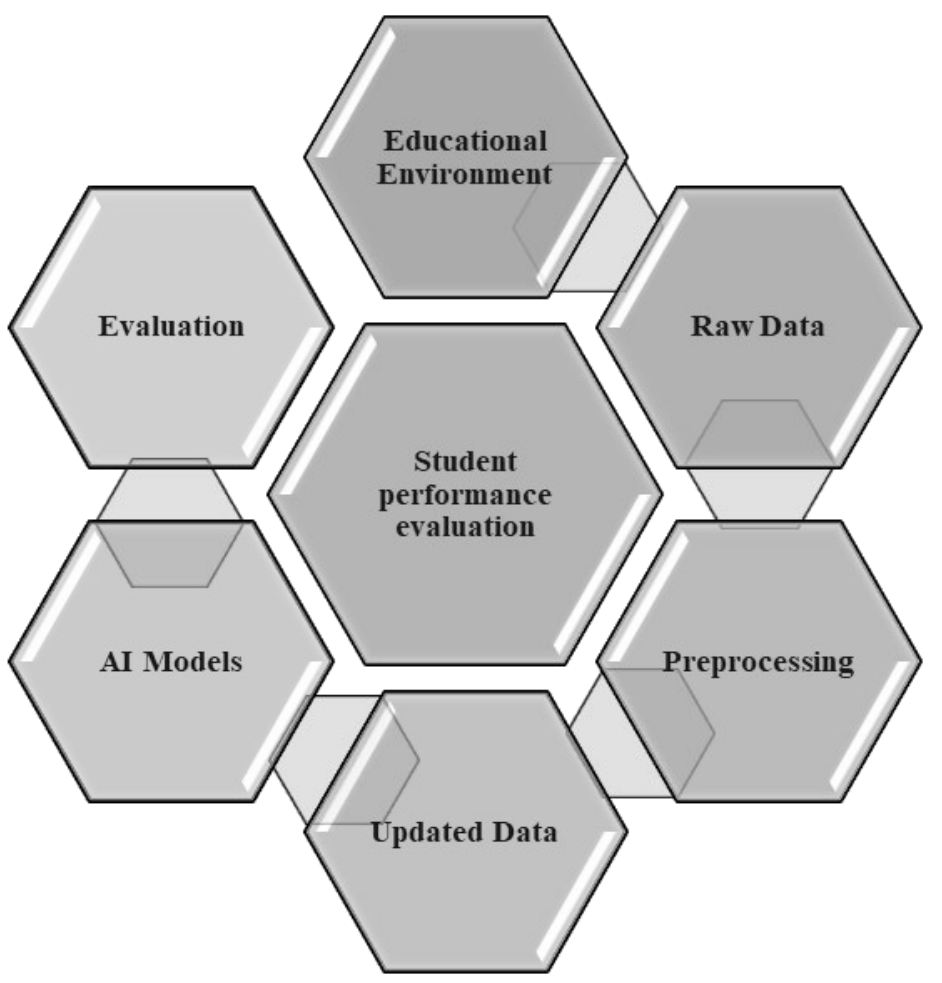

Figure 1. Student Performance Analysis

As shown in the Figure.1. To enhance the design process and prepare strategies for instructional support and instruction on the students' education, there is a great need to anticipate potential student behavior [9]. Data Mining methods analyses datasets and collect information to turn them into understandable structures for subsequent use. Machine Learning (ML), Collaborative Filtering (CF), Recommending Systems (RS), and Artificial Neural Networks (ANN) are the essential computational techniques that process this knowledge to predict the performance of the students, their grades, or the risk of dropping out [10-12].

Artificial intelligence has widely been used in data science applications to analyze dynamic interactions to cope with such limitations. It's able to learn without specific coding instantly. A Deep Neural Network (DNN) model, although it has a long-developed history in computing and data science, is attracting the interest [13] and implementations of a wide variety. DNN extends the ability to analyze complex data sets that conventional statistical methods cannot easily explain. It may establish indirect non-linear relations between variables of the study [14]. DNN has gained wider recognition and proven to be a great success in applying trend detection, classification, prediction, and forecasting in hospitals, environment, landscape, financial markets, etc. [15-17].

The use of DNN in the field of education science, furthermore, is limited. It can be attributed to the complexity of the modeled network, the difficulties in presenting an appropriate explanation (the black-box nature) for a modeled DNN structure, the time is taken for neural network training, and proneness over-fitting [1820]. In this paper, an area of expertise is compromised with both statistical learning and DNN research to mitigate the limitations by applying DNN. Initially, the educational data is evaluated with traditional data analysis, and statistical output confirmation is utilized to execute DNN planning, testing, and validation to constructing the DNN model with good configuration to classify and predict the students' results reliably [21, 22].

This study presents the students with an investigative analysis and examination of performance. The HDNN model aids as a useful standard educational tool that assesses student progress across universities, tackle certain inequality, and gradually improves education 4.0. Deep Learning is a set of methods that gives computers the capability to learn without human programming intervention. The DNN model assists as a prevailing quality educational tool that assesses the students' performance throughout academies, addresses differences, and, therefore, continually enhances education quality.

\section{Related works}

Ciolacu et al. [16] introduced a Machine Learning technique (MLT) to analyze Educational behavior that is rapidly going online, and the contents of the course are accessible in digital format. It allows for data analysis and data usage to evaluate learning processes. Active and engaging students' participation leads to a higher learning level for the 4th Revolution in Education. Machine learning methods have recently taken remarkable 
strides in the advancement of using data processing and predictions. Thus, it is much less used to measure the level of learning.

In Education 4.0, Ciolacu et al. [17] proposed an innovative method for supporting AI. The first involvement in the AI-assisted Higher Education System (AIHEF) with intelligent sensors and wearable devices for self-regulated learning. Furthermore, they clarify the initial outcomes of the didactic approaches of education 4.0 applied with machine learning algorithms and learning analytics. This case study is intended to estimate the students' ultimate scores before taking part in the final test.

Bagustari et al. [18] implemented LMS (Learning Management Systems) to support their operation. The adaptive user interface (AUI) has a crucial role in maintaining students involved in learning experiences in multiple technology changes. It is very important to investigate AUI's development in education 4.0 to understand its major experiment on this level. This study explores the AUI within the learning model suggested and applied by other researchers. When analyzing their methods, we use a qualitative perspective between the technology and the pedagogical element.

Analysis in Educational Data Mining (EDM) and Learning Analytics (LA) has developed as essential research fields that reveal valuable information from educational datasets for many uses, such as predicting students' progress. In modern educational environments, the ability to anticipate a pupil's success may be useful for behavior [19]. Current approaches have often been related to academic success, family income, and family assets, whereas family expense features and personal details of students are typically ignored. Efforts are made to examine the quality mentioned above sets by gathering data from various universities from the scholarship holding students.

Guo et al. [20] developed the federated learning-based educational data analysis framework FEEDAN, in which several institutions can create federations of education data analysis. None of them need to directly share the data of their students, and they still keep the data in their location to maintain their students' safety. They apply their methodology to evaluate two real educational datasets across two different federated paradigms of learning. The experiment findings show that it protects the privacy of the students and violates the limits of the data island by reaching a higher level of the analysis.

To overcome these issues, in this paper, Hybridized Deep Neural Network (HDNN) to predict student performance in Education 4.0. The educational information will be studied primarily with traditional statistical exploration. The validation of the statistical output will be utilized to execute Deep neural network validation, training, and testing to improve the DNN model with appropriate configuration backgrounds to precisely forecast and classify the students' performance.

\section{Hybridized Deep Neural Network (HDNN)}

In this study, Hybridized Deep Neural Network (HDNN) to predict student performance in Education 4.0. Education 4.0 is a term given to a link between new and comprehensive technologies, which in many ways, is estimated to have an impact on higher education. Robotics, mixed reality, and hyper-connectivity all play a part, and the most headlines have been artificial intelligence, fostered by large data and machine learning. AI could learn a student's routine at an effective appraisal of the day and remind them of its goals. It could analyze gaps in their academic performance and suggest corrective actions. Education 4.0 characterized by virtual courses involving an interactive occurrence in the practice of AI-driven and Blended Learning educational technology: personalized learning process, game-based learning using Augmented Reality/Virtual Reality (AR/VR), adaptive technologies, communities of practice, learning analytics, E-Assessment and intelligent Chabot. The demand for an education revolution is growing. Recently, research in AI has demonstrated impressive developments, leading to many AI-based technologies into machine learning in the industry. From medicine to transport, manufacturing, and education, the future of everything is shaped by AI. Machine learning is part of AI, allowing new knowledge and models to be extracted from data that have huge potential for benefit. Artificial intelligence in education is key to identifying new drivers for student performance and early disengagement, personalizing learning, answering basic questions of students, using learning analytics, and providing predictional modeling. Education 4.0 refers to the usage of technology in learning and teaching environments such as schools and universities. This is due to technology that enables the human-machine interface to look universal, subsidizing a fast uprising in innovation. This is to make graduates for future work and life; therefore, education 4.0 is very necessary. There is an increasing need for an education revolution. Recently, research in AI has seen remarkable advances that contribute to implementing the industrial machine learning of various AI-based technologies. From the medical world to logistics, development, and preparation, the future of all is AI shaped. Machine learning is part of AI, enabling the extraction of new information and models from data with tremendous potential. 


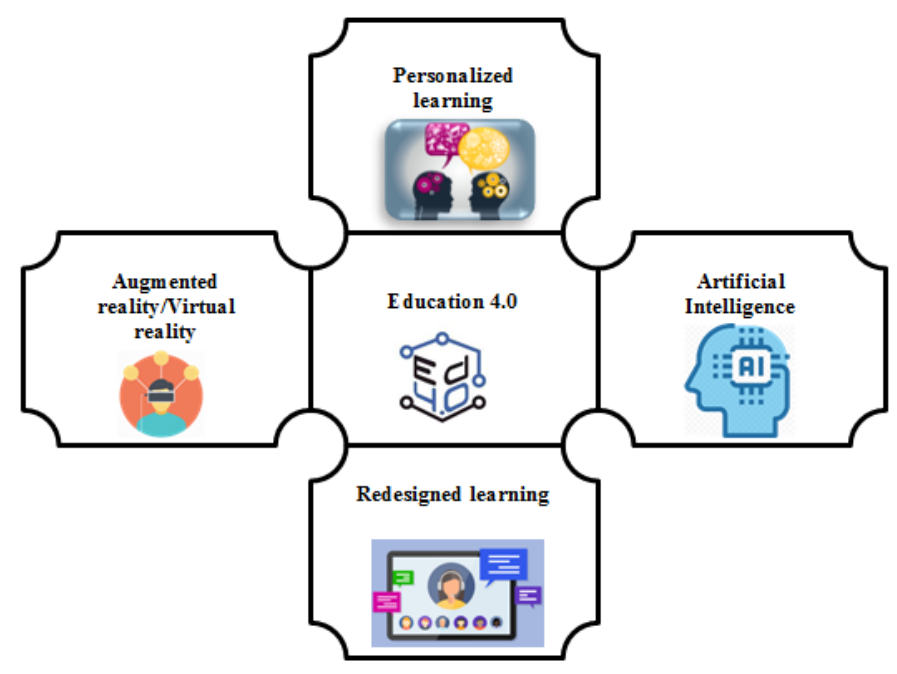

Figure 2: Education 4.0 framework

Figure 2 shows the education 4.0 framework. Education 4.0 completes the digital integration phenomenon in everyday life, where people, tools, and materials are aligned in solvent solving, debugging, and discovering new innovative theory. In education 4.0, there is ubiquitous information and a dynamic teaching and learning process. It is not hard to imagine what education 4.0 holds for us in developing technological advancements. The economic usage of information can considerably change the future of education 4.0. Economy 4.0 has brought us into an era of unprecedented transformation. In this period, the acceleration of change has increased dramatically. It's the virtual world that connects with the cyber world and blends into the real world. It is an era of innovative knowledge, although it is obsolete, and new knowledge comes into use. In education 4.0, the guidelines are a flipped classroom method. Interactive learning takes place in the classroom, and theoretical elements should be learned outside the classroom. The learner experiences no time-limited learning here. Even the learning is individually personalized and is carefully administered by an individual student. If a student develops a certain level of understanding, a high-level learning task is introduced. Besides, the instructor uses a rigorous practice of the given task until the master's level is reached. To support a positive learning experience in education 4.0, positive reinforcement is primarily used. A training course's learning outcomes are set by the institutions/government bodies in the teaching/learning process (and curriculum design). Students can still choose their preferred learning tools. For academic educators, education 4.0 is essential to remain agile and adapt to the changes necessary. The students must be prepared in line with industry requirements, in line with the fundamental principles of education 4.0. In the curriculum/learning process, the learning effects of a training course are specified by the institutions/government agencies. Education 4.0 is essential for academic educators to be versatile and adaptable to the necessary changes. Students must be trained according to business demands in compliance with basic education standards 4.0 .

Academically, both student's and teachers' main goal is to achieve maximum pass rates. Students who pay attention and participate in the classes can normally pass their exams. The time they spend and the ability to reason are essential. However, students have always had more struggles, and, in most cases, this fact is connected to a series of social attributes that influence students who are poorly performing. Academic students' performance has always been the main element in identifying a student's career and the Institutions' esteem. Thus, the suggested system supports to study the student "s as well as teachers' overall performance and recommend proper training of the teachers. Deep Learning is a set of methods that gives computers the capability to learn without human programming intervention. The educational information will be studied primarily with traditional statistical exploration. The validation of the statistical output will be utilized to execute Deep neural network validation, training, and testing to improve the DNN model with appropriate configuration backgrounds to precisely forecast and classify the students' performance. The DNN model assists as a prevailing quality educational tool that assesses the students' performance during academies, tackling differences and, therefore, continually enhancing the quality of education. This enables students to monitor their performance and to evaluate whether they can perform correctly in examinations or not. Teachers are mainly involved in the system. The prediction tool looks at the result of the teachers. The students' performance is improved, and the teacher helps them through effective decision-making. There is an obvious relationship between participation rate and passing the examination. The Neural Network is trained with data to help it classify behavior patterns. This analyses the initial dataset and 
reduces the characteristics that lead to students who do not pass exams to a minimum. Finally, the previously qualified network is used to decide whether students will pass their exams by changing the parameters.

The predicting task of student performance can be expressed as a sequential event prediction issue, given a past event sequence $u \triangleq\left(u_{1}, \ldots u_{T}\right)$ taken by a student, determining the probability of future event sequence $x \triangleq\left(x_{T+C, \ldots} x_{T}\right)$ where $C \in \mathbb{Z}_{+}$.

In the form of online classes, every event $u_{t}$ denotes a student's action linked with a time stamp. In other words, $u_{t}$ is stated as a paired tuple of $\left(b_{t}, c_{t}\right)$. Every action $u_{t}$ denotes, for instance, a quiz answered correctly/incorrectly, or a lecture video viewed or project submitted and passed/failed and $c_{t}$ denotes the respective timestamp. Academically, both student's and teachers' main goal is to achieve maximum pass rates. Students who pay attention and participate in the classes can normally pass their exams. The time they spend and the ability to reason are essential. However, students have always had more struggles to a series of social attributes that influence poorly performing students. In online classes, every event of the student's action is linked with a time stamp.

The log-likelihood of $q(x \mid u)$ can be expressed in equation (1), given fixed-dimensional embedding representation $o$ of $u$,

$$
\log q(x \mid u)=\sum_{j=T+\varepsilon}^{T^{s}} \log q\left(x_{j} \mid o\right)
$$

As inferred from the equation (1) where $\sum_{j=T+c}^{T^{s}} \log q\left(x_{j} \mid o\right)$ Denotes the individual log-likelihood, and those estimated scores can be added up to estimate long-term student outcomes.

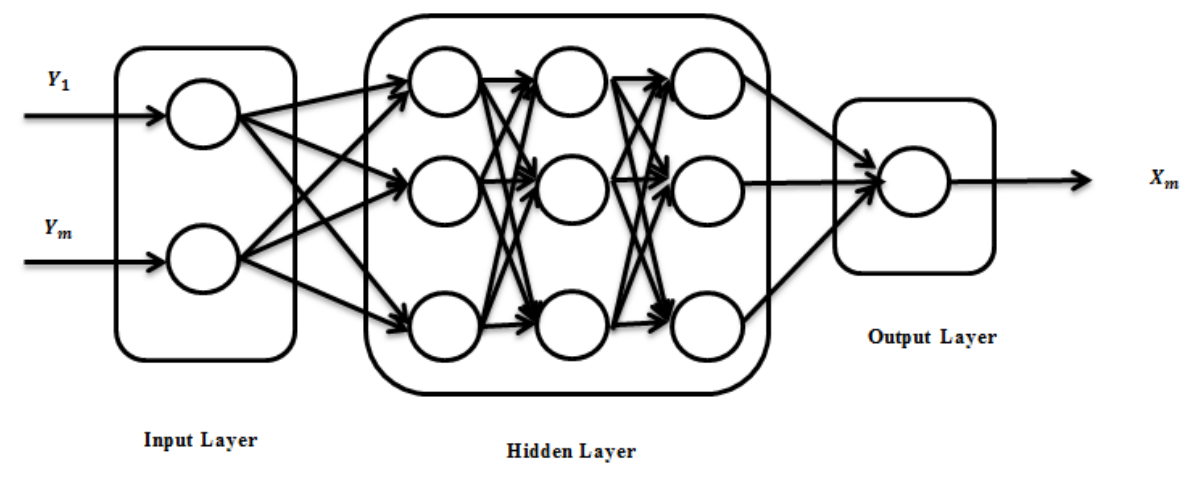

Figure 3: Deep neural network architecture

Figure 3 shows the deep neural network architecture. Deep learning methods are designed to learn hierarchies of attributes with a higher level attribute that combines other low features. Multiple approaches for deeper and higher architectures are learned. A deep neural network is a multi-model NN class. Model of layers of input, hidden layers of an arbitrary number, and a layer of output. The layers consist of neurons that share similarities with the brain neurons of the human.

The neuron is a non-linear function that maps input vectors $\left\{J_{1, \ldots} J_{m}\right\}$ to an output $X$ via a weighted vector $\left\{s_{1}, \ldots, s_{m}\right\}$ and to a feed-forward function $f$ expressed as,

$$
\left.X=f\left(\sum_{j=0}^{\|} s_{j} J_{j}\right)=f\left(s^{\mathrm{t}}\right]\right)
$$

An input layer collects data, and the input layer is fed to the hidden layer. The output layer of ith neuron $x_{\tilde{i}}$ of vector $\hat{X}$ are evaluated employing the sum of weighted input attributes $y$ and $s$,

$$
x_{i}=\theta\left(\sum_{j=1}^{M_{j}} s_{j i} y_{j}\right)
$$

As shown in equation (3) where $\theta$ is the activation function, $y_{j}$ is the output layer from the prior layer of $j$ th neuron and $M_{j}$ is the overall number of $j$ thlinking lines to the ith neuron. This activation function provides all 
positive values to pass via, while negative values are allocated 0 . The activation function $\theta$ is utilized to transmit the sum of weighted inputs to the output layer. The consequential activated node for the subsequent input layer is thus,

$$
y_{i}=\theta\left(x_{i}\right)
$$

The objective of the model is to optimize the weights $s$ such that squared loss error is decreased. This can be attained by utilizing stochastic gradient descent. It iteratively updates the weight vector, which definitively aims to direct to the least gradient of the loss function. To estimate a stochastic gradient descent update equation,

$$
s^{\text {new }}=s^{\text {old }}-m \cdot(X-t) \cdot X(1-X) \cdot J
$$

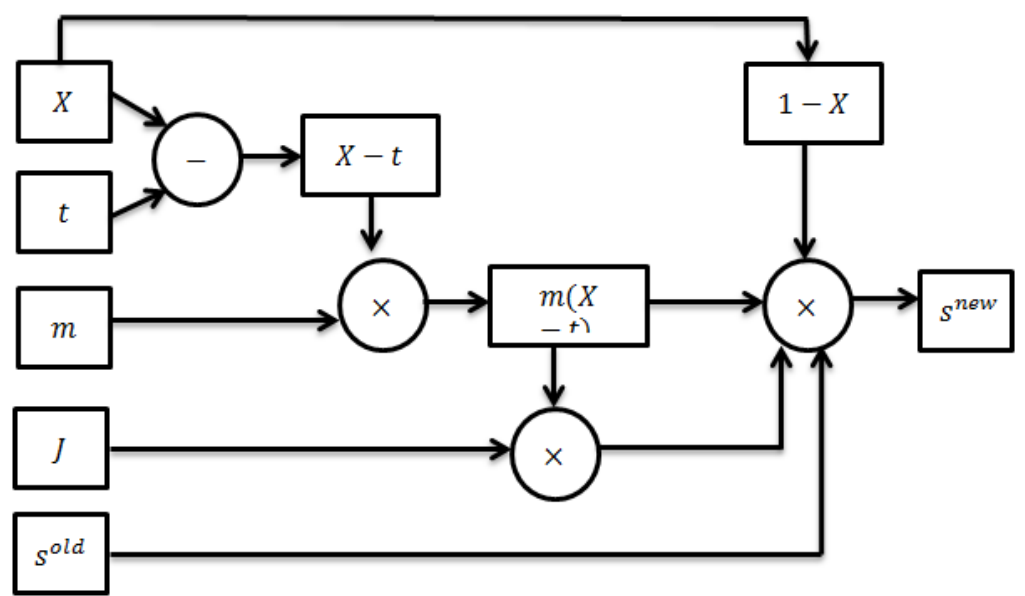

Figure 4: stochastic gradient descent update

Figure 4 and equation 5 shows the stochastic gradient descent update. This research aims to improve the pass rate of studies among students by developing and evaluating a computerized learning model. To enable it to identify behavior patterns, the Neural Network is trained with data. The original data set is then examined, and the attributes contributing to the pass rate of students who do not pass their examinations are reduced to a minimum. Finally, the previously trained network is being utilized to forecast whether students can pass their exams with the parameters' changes. The purpose of this research is to enhance student pass rates by designing and testing an IT model. The Neural Network is trained with data to help it classify behaviour patterns. This analyses the initial dataset and reduces the characteristics that lead to students who do not pass exams to a minimum.

The error function $G$ of computed feed-forward or backpropagation based deep neural network is evaluated as the sum of the square variance between the target values and desired output,

$$
G=\frac{1}{2} \sum_{i}^{M_{i}}\left(x_{i}-t_{i}\right)^{2}
$$

As derived in equation (6) wheret $t_{i}$ is the target value of neuron $\tilde{f}$ in the output layer and $M_{\mathrm{i}}$ is the overall number of output neurons. 


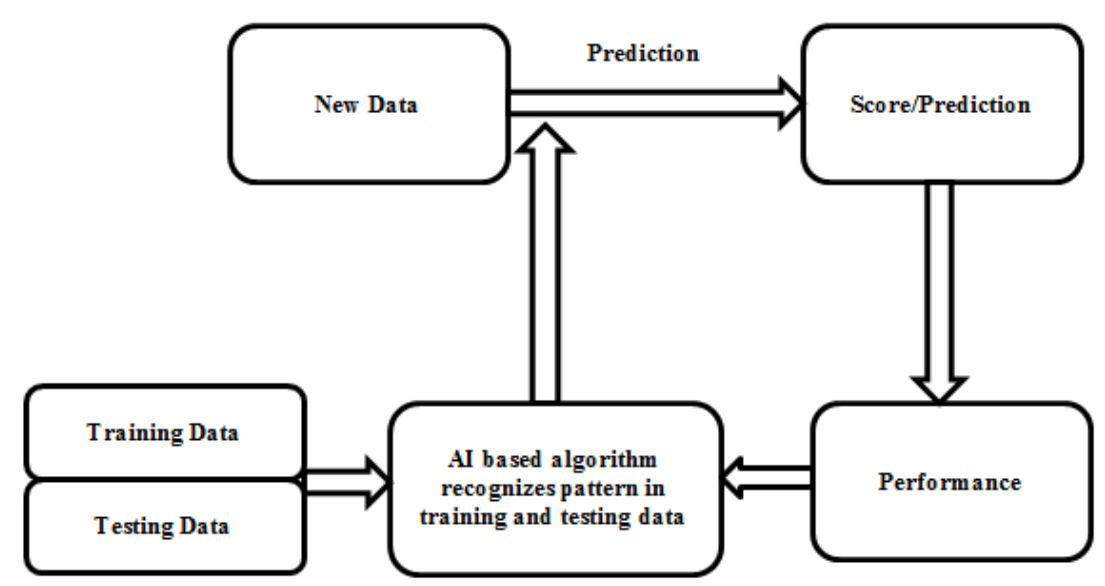

Figure 5: Deep learning process

Figure 5 shows the deep learning process. This research's major objective is to improve learning quality by tracking students and predicting the performance of the students in the education 4.0 environment. To assess the performance of DNN, this study presents many different standpoints that alleviate the rising of over-fitting problem to guarantee the correctness of DNN performance. The DNN model aims to measure students' success in academia, resolve discrepancies, and ultimately increase the standard of education. This makes it easier for students to track their performance to decide whether they will administer exams correctly or not. The instructor outcomes are analyzed in the prediction tool. The success of the pupils is enhanced, and the teacher supports them in making successful decisions. Hence, there is a direct connection between the participation rate and the test.

The assessment involves the calculation of the confusion matrix and the Mean Square Error. A welltrained DNN model might have a less mean square error value, which denotes that the forecasted outputs congregate close to the target outputs $t_{j \mathrm{j}}$.

$$
\text { MSE }=\frac{1}{M_{j} M_{i}} \sum_{i=1}^{M_{i}} \sum_{j=1}^{M_{j}}\left(x_{j i}-t_{j i}\right)^{2}
$$

As discussed in equation (7), the trained network over-fitting is probable if the determined mean square error value is small. This additional specifies that DNN works better in training, testing phase, and validation. An Epoch is one backpropagation and one feed-forward. Every epoch supports decreasing the cost function. In a

DNN, an epoch is iterated $m$ th times, optimizing and updating the gradients. DNN is a deep learning (DL)structure that provides operational models composed of many hidden layers to learn different data depictions with multi-level abstraction. DL has a tremendous ability to self-adapting and self-learn, making it widely deliberate and effectively tackling complex real-world issues. The results are evaluated based on given input data, expected results, and characterization of outputs and other factors. The accuracy of the prediction is important for evaluating performance. The goal is to determine whether students will pass or break their final exams based on all the parameters analyzed. A study should be carried out of students who failed their test, the main parameters that are contributing to their low performance, and how those parameters can be improved.

\section{Experimental Results and Discussion}

Student performance assessment is essential for evaluating the viability of the development. Daily check not only increases the student's performance and helps to clarify where the student is missing. Completing the assessment process requires much manual effort since even one college may involve thousands of students. Hybridized Deep Neural networks (HDNN) have been used for psychometric factors to predict the students' performance. A psychometric factor is known as student motivation, study behavior, time for interaction, and family support. These attributes used to make a system look very clear, user-friendly, and simple. It helps the professor to determine the performance of the students based on their preferences and behavior. However, most researchers seldom extend these characteristics in forecasting student performance because it relies mainly on qualitative data, and it is sufficient to collect reliable data from respondents. Predicting student performance helps instructors and learners improve the experience of learning and teaching. Figure 6 demonstrates the student performance ratio analysis of Proposed HDNN and other traditional methods. Hybridized HDNN networks were 
used to model the success of the students in psychometric factors. A psychometric aspect is called student inspiration, research, contact time and family support.

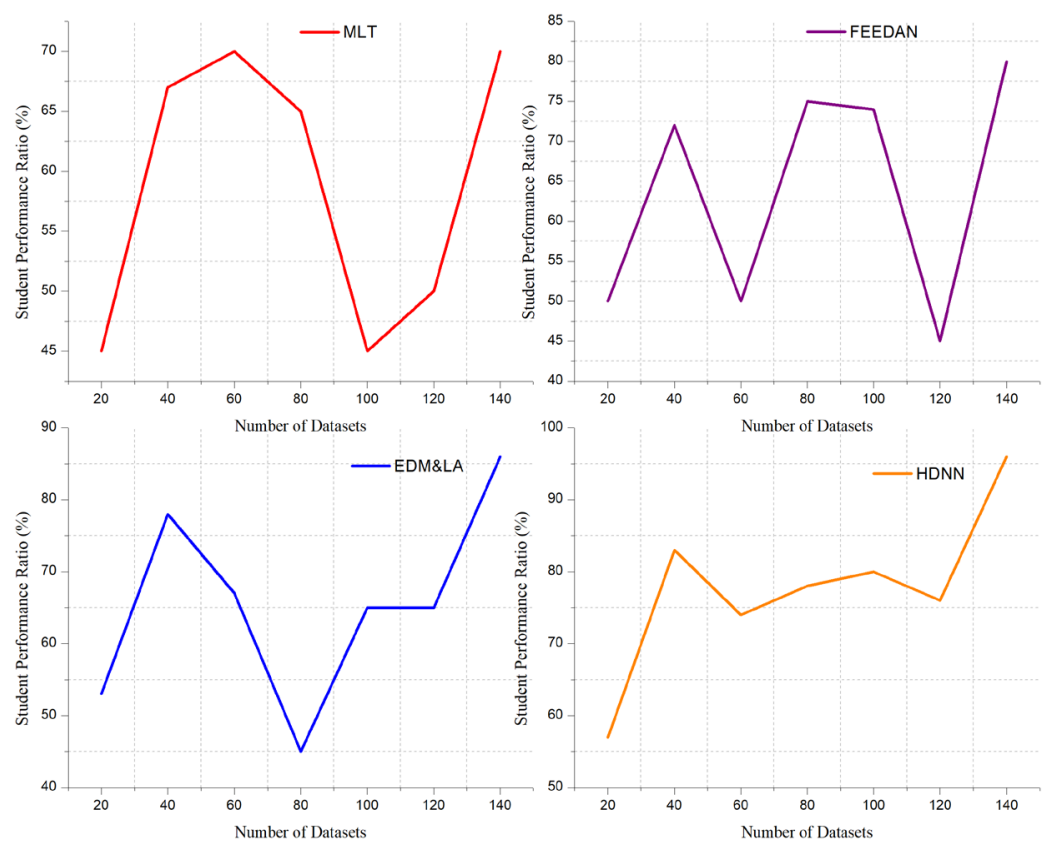

Figure 6. Student performance analysis

DNNs have been applied to different estimation methods, virtually by analyzing students' evaluation performance, as seen in the following cases. An HDNN is qualified to predict scores of assessments taking partial scores into account during the course. An HDNN using Cumulative Grade Point Average has predicted student success. HDNN models have been compared to identify the best one for predicting student academic performance. Finally, the ability of HDNNs to predict academic achievement is compared with the other existing traditional approaches. Besides, educators may track the accomplishments of their students. Students may increase their learning activities, encouraging the administration to improve the efficiency of the systems. Simultaneously, the application of HDNN strategies for different entities may be focused on specific needs. Figure 7 demonstrates the student prediction ratio analysis of Proposed HDNN and other traditional methods. For performance assessment, the exactness of the forecast is important in education 4.0. It is supposed to decide whether a student fails or interrupts his final examinations, depending on all the criteria' interpretation. Besides, students who have failed the exam, the principal parameters that lead to their poor results, and how these parameters may be strengthened, should conduct a report. 

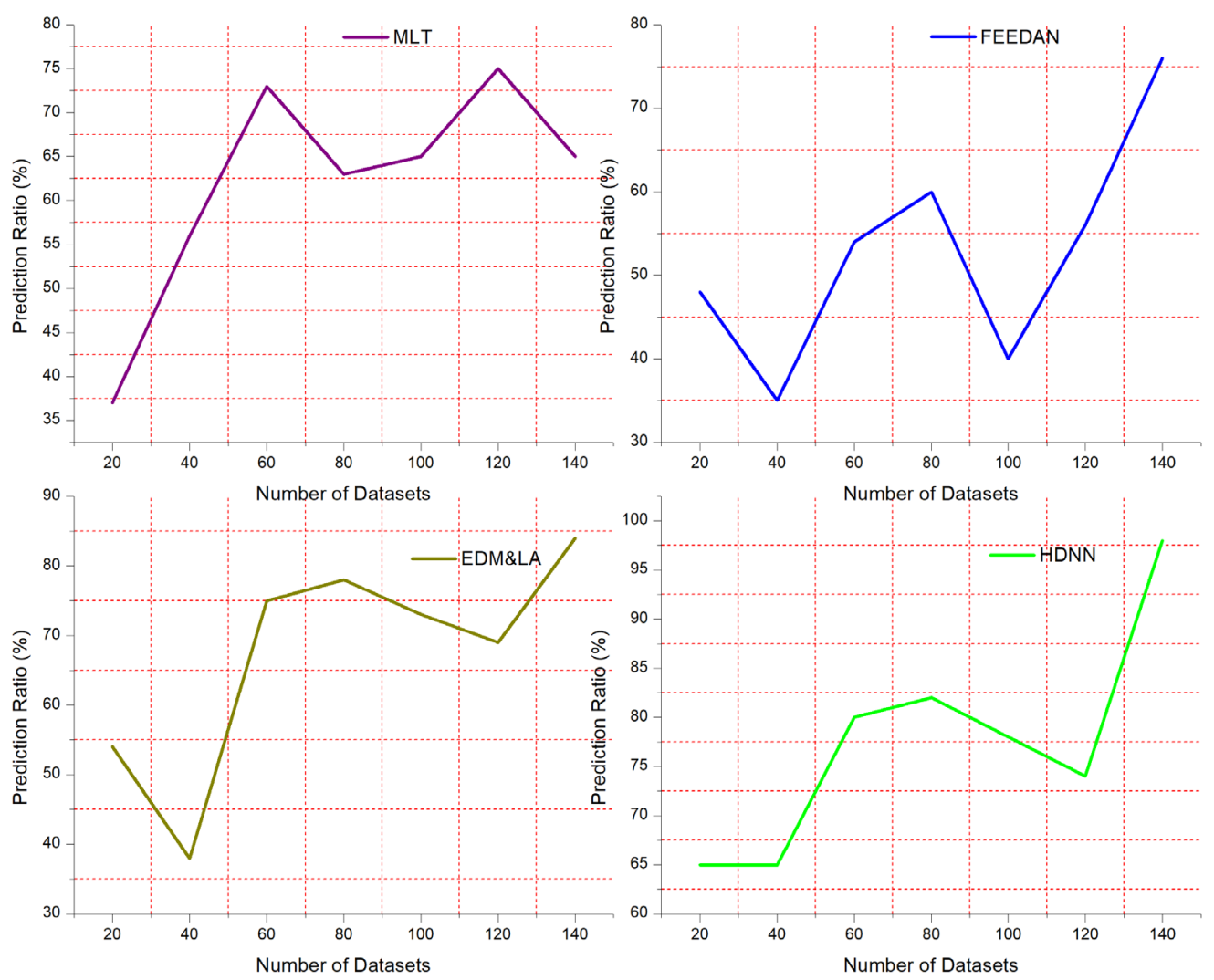

Figure 7. Student Prediction Ratio Analysis

A platform based on artificial intelligence that considers HDNN as being one of the methods of artificial intelligence reduces learning disorientation and overloading cognitive problems of the students. The HDNN performed well in the process as compared to other implemented learning models. Besides, a DNN is applied to enhance the teaching system's efficiency, and the DNN result can satisfy the viability and accuracy criteria of the system. This study would rely on data samples from a public student to guarantee the predictability of academic performance. The HDNN model will assist as a structure and guide for forecasting prospective students' academic performance and thus resolving certain challenges that have an ongoing impact on student learning progress, and hence on the quality of education. Besides, HDNN with balanced data sets can provide depressingly high predictability. Accuracy is essential as it can be instrumental in designing educational initiatives to improve the teaching-learning outcomes, save time and effort for government resources and educators. Figure 8 demonstrates the student prediction ratio analysis of Proposed HDNN and other traditional methods. 

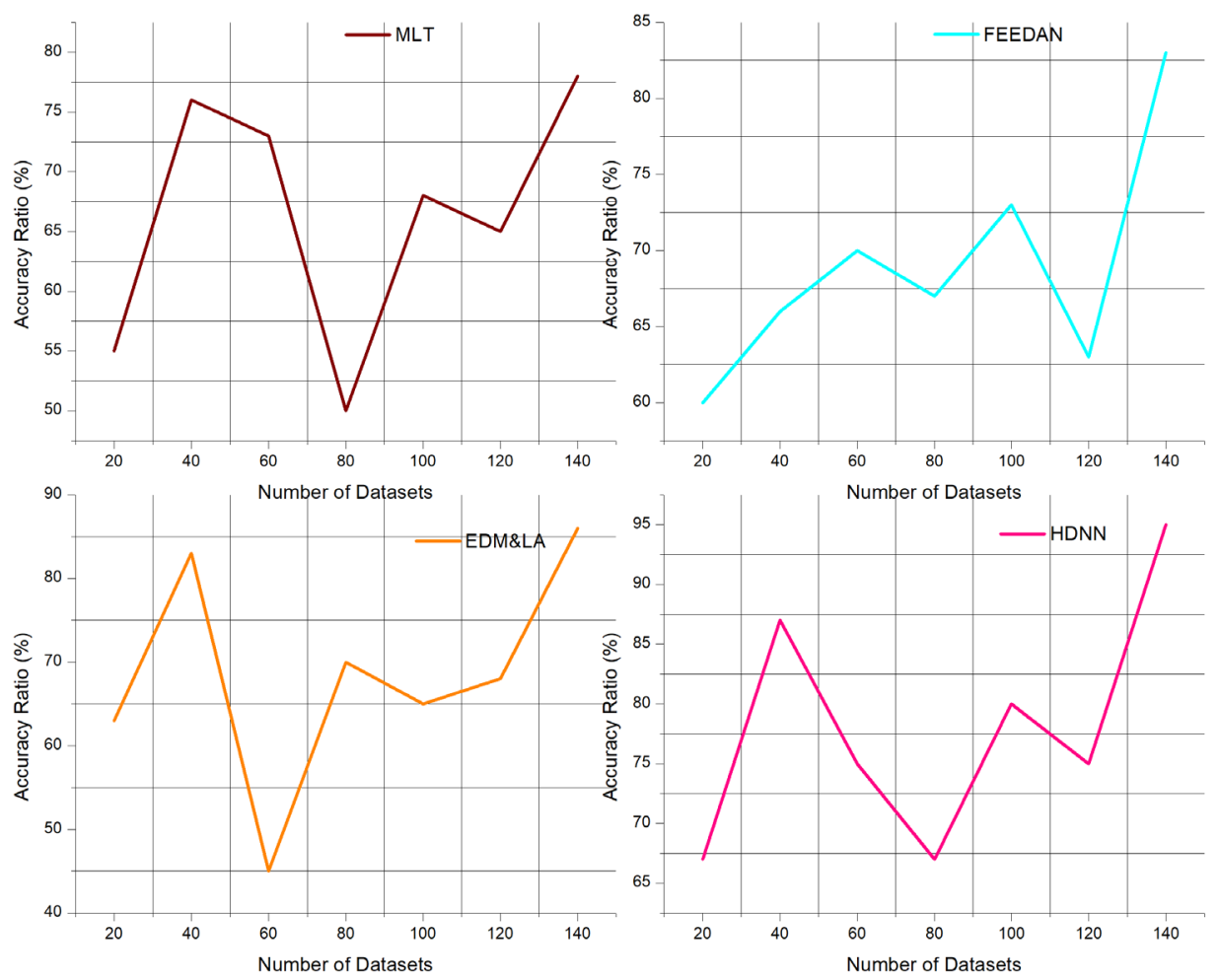

Figure 8. Accuracy Evaluation

HDNN can be used on a regular, ongoing basis to assess student learning. These evaluations reveal how and what students are progressing and educate the next steps in teaching and learning during the course. Rather than asking students if they understand or have any questions, it might be more structured and comprehensive to ask students at the end of the class to edit the most relevant aspects of the most challenging part of the test used to review the lecture. Collecting and analyzing the answers offers an insight into what ideas students have learned and the next teaching step. Providing students with input on these topics gives them insight into their learning. The proposed HDNN effectively analyzes student knowledge for student performance analysis. Figure 9 shows the Student Knowledge Analysis of proposed HDNN with other traditional methods. 

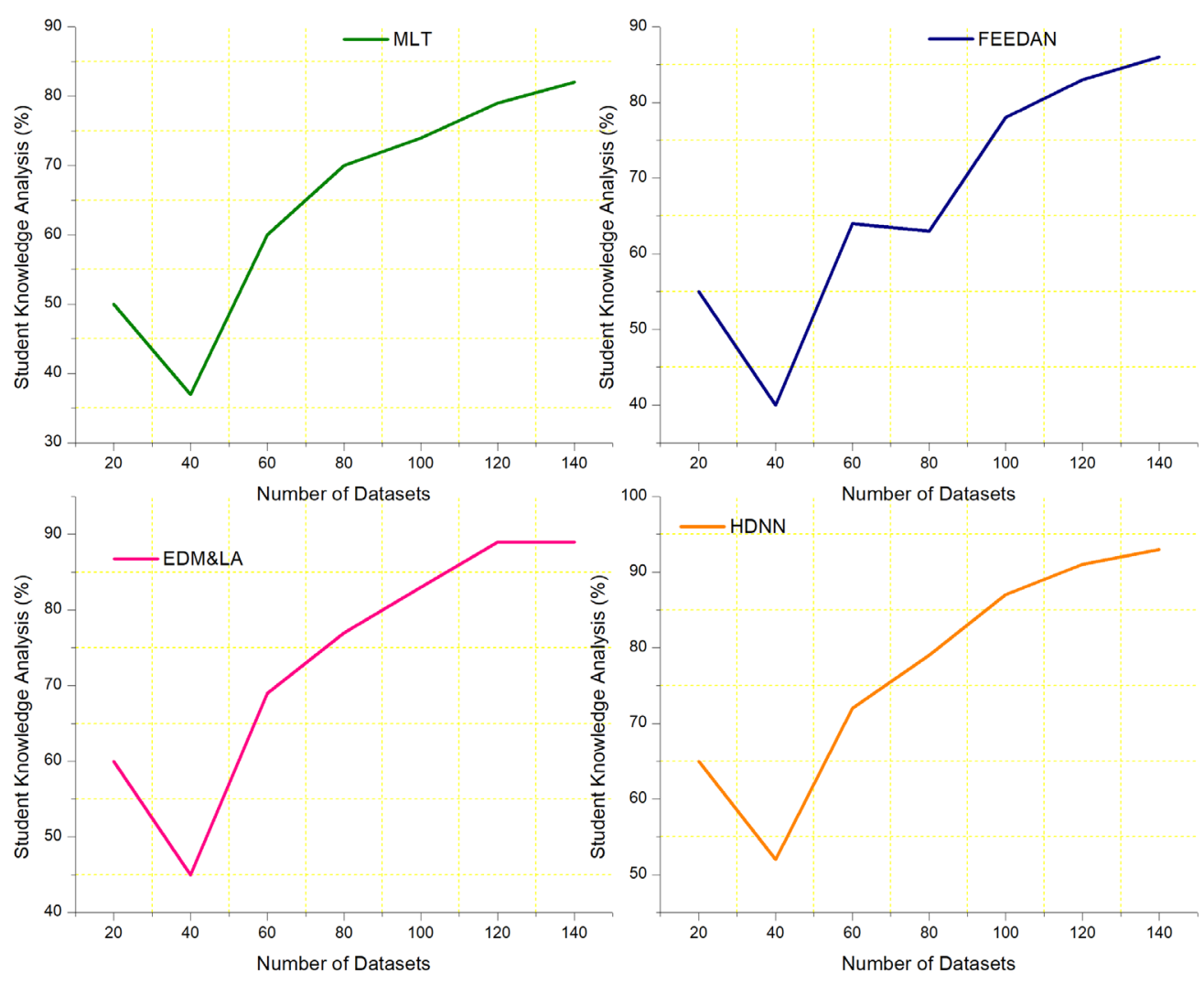

Figure 9. Student Knowledge Analysis

Student engagement is one good academic idea that is attracting growing recognition globally. It is seen as a significant factor in determining and enhancing the standard of undergraduate education. Student engagement is the level of attention that students devote to educationally propositional tasks that specifically contribute to their learning and growth. It happens when students make a stronger attachment in education that focuses not only on results as a structured measure of progress but also on understanding and integrating the matte subject. The more students engage in their research, the better they get to understand what they are studying. They become to handle uncertainty, accept ambiguity, and collaborate with individuals with diverse cultures or different viewpoints. The impact of student engagement on academic quality in higher education and student growth and learning is evident in the development of many evaluative tools in undergraduate education. Figure 10 demonstrates the student engagement ratio analysis of Proposed HDNN and other traditional methods. Our mission is to recognize students at risk in the exams and encourage teachers to directly boost their academic performance directly via e-mails. This study revealed that a deep neural network could operate with even less data, taking in-depth knowledge of the model's dataset. Furthermore, a DNN was proposed to predict a behavioral model that would enhance student success. 

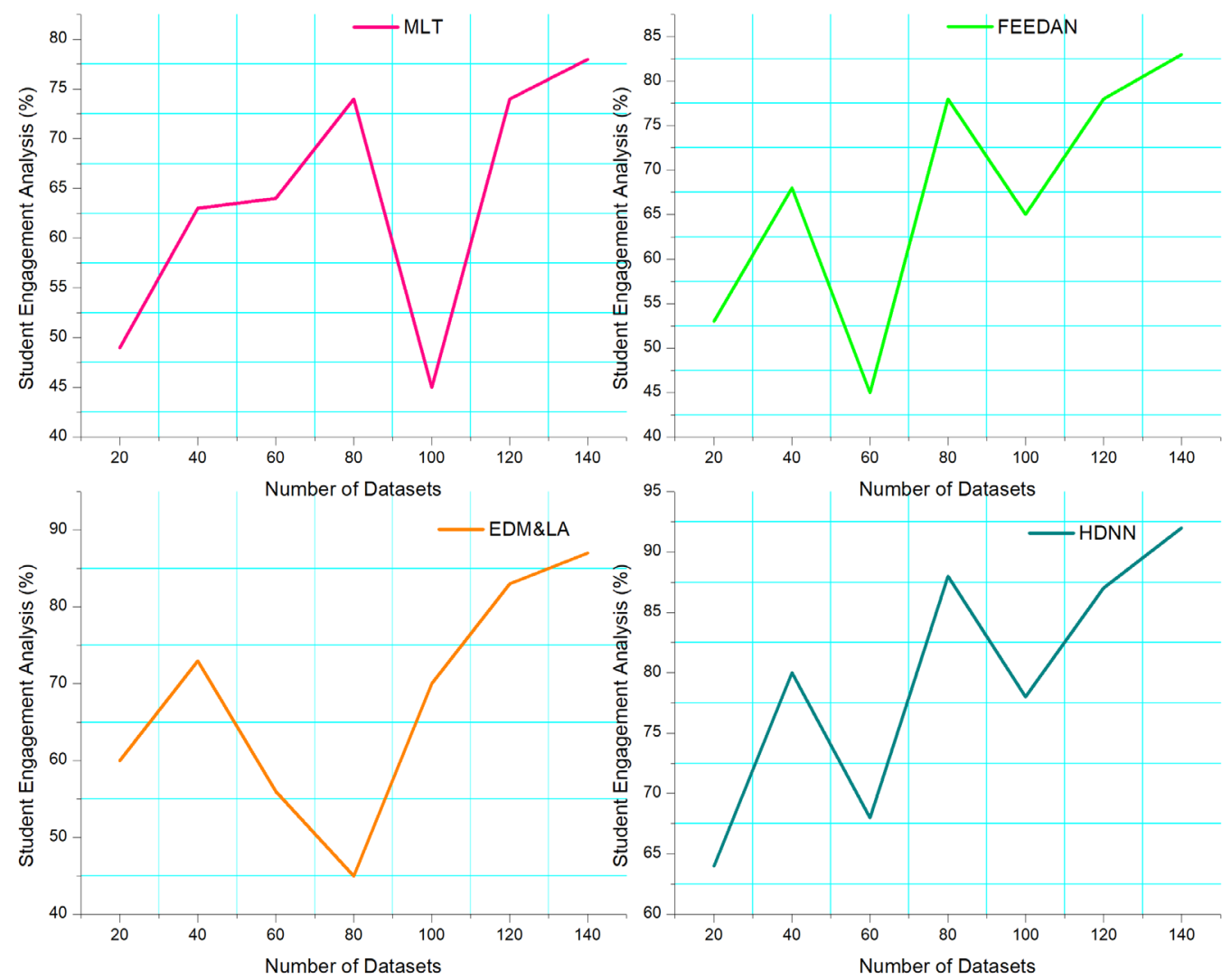

Figure 10. Student Engagement Ratio

The proposed Hybridized Deep Neural Network (HDNN) to predict student performance for education 4.0 achieves a high prediction ratio in terms of students' performance forecasting and accuracy when compared to other existing Machine Learning technique (MLT), Educational Data Mining (EDM), and Learning Analytics (LA), federated learning-based educational data analysis framework FEEDAN methods.

\section{Conclusion}

This paper presents the Hybridized Deep Neural Network (HDNN) to predict student performance in Education 4.0. We aim to determine students at risk early in the exams and personally motivate them from instructors with mails to improve their academic success. Through experimentation, this study found that a deep neural network can perform better even with fewer amount of data by taking profound knowledge about the dataset on the model. Besides, the utilization of a DNN has been suggested to forecast a behavioral model that enhances academic performance. A deep neural network can attain greater accuracy and will outperform other existing methods. This proposed system is reliable and can aid in forecasting thestudent's performance and determine students who have a higher unintended of failing beforehand to offer a remedy.

\section{Acknowledgments}

This study was supported by the Natural Science Foundation of the Jiangsu Higher Education Institutions of China(No. 17KJD520006).

\section{References}

[1]. Ciolacu M, Svasta PM, Berg W, Popp H. Education 4.0 for tall thin engineer in a data driven society. In2017 IEEE 23rd International Symposium for Design and Technology in Electronic Packaging (SIITME) 2017 Oct 26 (pp. 432-437). IEEE 
[2]. Almeida F, Simoes J. The role of serious games, gamification and Industry 4.0 tools in the Education 4.0 paradigm. Contemporary Educational Technology. 2019 Apr 16;10(2):120-36.

[3]. Xu X, Li D, Sun M, Yang S, Yu S, Manogaran G, Mastorakis G, Mavromoustakis CX. Research on key technologies of smart campus teaching platform based on 5G network. IEEE Access. 2019 Jan 22;7:20664-75.

[4]. Ciolacu MI, Binder L, Svasta P, Tache I, Stoichescu D. Education 4.0-Jump to Innovation with IoT in Higher Education. In2019 IEEE 25th International Symposium for Design and Technology in Electronic Packaging (SIITME) 2019 Oct 23 (pp. 135-141). IEEE

[5]. Abdel-Basset M, Manogaran G, Mohamed M, Rushdy E. Internet of things in smart education environment: Supportive framework in the decision-making process. Concurrency and Computation: Practice and Experience. 2019 May 25;31(10):e4515.

[6]. Lopez-Garcia TJ, Alvarez-Cedillo JA, Sanchez TA, Vicario-Solorzano CM. Review of trends in the educational model of distance education in Mexico, towards an education 4.0. Computer Reviews Journal. 2019;3:111-21.

[7]. Kadry S, Roufayel R. How to use effectively smartphone in the classroom. In2017 IEEE Global Engineering Education Conference (EDUCON) 2017 Apr 25 (pp. 441-447). IEEE.

[8]. Shakeel PM, Baskar S. Automatic human emotion classification in web document using fuzzy inference system (FIS): human emotion classification. International Journal of Technology and Human Interaction (IJTHI). 2020 Jan 1;16(1):94-104.

[9]. da Motta Reis JS, Costa AC, Espuny M, Batista WJ, Francisco FE, Gonçalves GS, Tasinaffo PM, Dias LA, da Cunha AM, de Oliveira OJ. Education 4.0: Gaps Research Between School Formation and Technological Development. In17th International Conference on Information Technology-New Generations (ITNG 2020) 2020 (pp. 415-420). Springer, Cham.

[10]. Yassine S, Kadry S, Sicilia MA. A framework for learning analytics in moodle for assessing course outcomes. In2016 IEEE Global Engineering Education Conference (EDUCON) 2016 Apr 10 (pp. 261266). IEEE.

[11]. Putra AB, Syafrudie HA, Yunos JM, Nidhom AM, Smaragdina AA, Sembiring AI. Analysis of the Necessity for Heutagogical Approach Through 4Cs Skills as Innovation for Vocational Lectures in the Education 4.0. In1st Vocational Education International Conference (VEIC 2019) 2019 Dec 19 (pp. $362-$ 371). Atlantis Press.

[12]. Selvakumar S, Inbarani H, Shakeel PM. A hybrid personalized tag recommendations for social e-learning system. International Journal of Control theory and applications. 2016;9(2):1187-99.

[13]. Nieto Y, García-Díaz V, Montenegro C, Crespo RG. Supporting academic decision making at higher educational institutions using machine learning-based algorithms. Soft Computing. 2019 Jun;23(12):4145-53.

[14]. Williamson B. Policy networks, performance metrics and platform markets: Charting the expanding data infrastructure of higher education. British Journal of Educational Technology. 2019 Nov;50(6):2794-809.

[15]. Mogoş RI, Bodea CN, Dascălu IU, Safonkina O, Lazarou E, Trifan EL, Nemoianu IV. Technology enhanced learning for industry 4.0 engineering education. Rev. Roum. Sci. Tech. Ser. Electrotech. Energy. 2018;63:429-35.

[16]. Alvarez-Cedillo J, Aguilar-Fernandez M, Sandoval-Gomez Jr R, Alvarez-Sanchez T. Actions to Be Taken in Mexico towards Education 4.0 and Society 5.0. International Journal of Evaluation and Research in Education. 2019 Dec;8(4):693-8.

[17]. Gaol FL, Napitupulu TA, Soeparno H, Trisetyarso A. Learning framework in the industrial age 4.0 in higher education. In2018 Indonesian Association for Pattern Recognition International Conference (INAPR) 2018 Sep 7 (pp. 227-232). IEEE.

[18]. Hong C, Ma WW. Applied Degree Education and the Future of Work.

[19]. Buckley SB, editor. Promoting Inclusive Growth in the Fourth Industrial Revolution. IGI Global; 2020 Jun 19.

[20]. Frolova VB, Kalugina OA, Artamonova LS, Boykov AI. Studying the practice of performance assessment and rating system implementation in higher institutions. International Electronic Journal of Mathematics Education. 2016 Aug 2;11(5):1185-93.

[21]. Saqr M, Fors U, Tedre M. How the study of online collaborative learning can guide teachers and predict students' performance in a medical course. BMC medical education. 2018 Dec;18(1):1-4.

[22]. Putra AB, Mukhadis A, Mahamad AK, Sembiring AI. Development of MOOCs synchronized life-based learning to improve the quality of outcomes in prospective vocational teachers in the era of education 4.0. InJournal of Physics: Conference Series 2020 (Vol. 1456, No. 1, p. 012051). IOP Publishing. 
[23]. Ciolacu M, Tehrani AF, Beer R, Popp H. Education 4.0-Fostering student's performance with machine learning methods. In2017 IEEE 23rd International Symposium for Design and Technology in Electronic Packaging (SIITME) 2017 Oct 26 (pp. 438-443). IEEE.

[24]. Ciolacu M, Tehrani AF, Binder L, Svasta PM. Education 4.0-artificial intelligence assisted higher education: Early recognition system with machine learning to support students' success. In2018 IEEE 24th International Symposium for Design and Technology in Electronic Packaging (SIITME) 2018 Oct 25 (pp. 23-30). IEEE.

[25]. Bagustari BA, Santoso HB. Adaptive User Interface of Learning Management Systems for Education 4.0: A Research Perspective. InJournal of Physics: Conference Series 2019 Jun 1 (Vol. 1235, No. 1, p. 012033). IOP Publishing.

[26]. Daud A, Aljohani NR, Abbasi RA, Lytras MD, Abbas F, Alowibdi JS. Predicting student performance using advanced learning analytics. InProceedings of the 26th international conference on world wide web companion 2017 Apr 3 (pp. 415-421).

[27]. Guo S, Zeng D, Dong S. Pedagogical Data Analysis Via Federated Learning Toward Education 4.0. American Journal of Education and Information Technology. 2020 Aug 4;4(2):56. 